\title{
Finite - element model for simulations of fully coupled thermomechanical processes in shape memory alloys
}

\author{
F. Richter ${ }^{\mathrm{a}}$, O. Kastner, G. Eggeler \\ Institute for Materials, Department of Materials Science, Ruhr-University Bochum, 44780 Bochum, Germany
}

\begin{abstract}
The Müller-Achenbach-Seelecke model for shape memory alloys is able to address full thermomechanical coupling of mechanical and thermal fields. The model roots in a stringent interpretation of thermodynamical principles and interprets the behavior as resulting from an interplay of austenite with two generic martensite twin variants. Its constitutive behavior covers both pseudoplasticity, pseudoelasticity and the characteristic shape memory effect upon temperature changes. Thus, the model reflects the complex, nonlinear hysteretic and thermomechanically coupled material behavior of SMAs on a physically sound basis.

In this contribution we investigate the capability of the MAS model in a typical engineering setting, viz. the shrink fitting of a SMA bushing onto a linear-elastic shaft. In this case, the shrink fitting is caused by a martensite-austenite phase transition of the bushing upon temperature change from low to high level (shape memory effect). To address all geometrical implications we employ a finite-element implementation of the MAS model into ABAQUS TM. The reliability of the FEM model is proven by comparison to the classical solutions for the linear-elastic and the ideally elastic-plastic case. The MAS model is implemented into ABAQUS using the UMAT interface. The results arrived at with this model are validated against the classical solutions and show the significance for the full thermomechanical coupling which becomes particularly evident in this setting.
\end{abstract}

\section{Introduction}

Shape memory alloys (SMA) are increasingly being employed as sensors and actuators. To assign them a function their behavior should be accessible to physical interpretation to utmost precision, hence reliable modeling techniques are mandatory. Depending on temperature, the constitutive behavior of SMAs is characterized by either pseudoplasticity (at low temperature) or by pseudoelasticity (at high temperature). Often sensitive machinery like sensors and actuators are equipped with SMA, requiring precise prediction regarding their performance under various mechanical and thermal loads. This thermomechanical interaction may not only be imposed by the intended use, but is also dictated by the inherent characteristic of thermomechanical coupling in SMA: as the composition is changing the percentage of austenite and martensite variants, temperature effects are caused by latent heat release. This effect entails a modification of the material properties while the externally applied loads progress. Hence, mechanical and thermal equations need to be solved simultaneously. This feature of fully thermomechanical coupling is captured by the model named after Müller, Achenbach and Seelecke, capable of reflecting all the characteristics of SMA [1-9]. Recently, a finite-element-method (FEM) implementation of the MAS model into the commercial software package ABAQUS (ABAQUS, Inc., Dassault Systèmes Simulia Corp., Providence, RI, USA) was elaborated at the Department of Materials Science of the Ruhr-University Bochum and tested upon pure pseudoelastic and quasiplastic settings [10]. In this contribution we apply the FEM-embedded MAS model to another important test case, the shape memory effect. Our scenario concerns the shrink fit problem of a SMA bushing which is widened at low temperature (where it is martensitic) so as to fit onto a linear-elastic shaft, see Fig. 1. The widening (radial and tangential extensions) cause quasiplastic defomations by de-twinning of martensite variants. When placed over the shaft, the shape memory effect is triggered by an increase of temperature. Once in contact, tangential transformation strains cause radial and hoop stresses both in the bushing and in the shaft.

In the conventional treatment of elastic shrink fittings the holding forces of the bushing are induced by thermal contraction. We proceed to explain the classical treatment of such problem for the case of an ideally elastic bushing (Sec. 2.1) and an ideally elastic-plastic bushing (Sec. 2.2). The according analytical solutions serve as benchmark for respective ABAQUS TM FEM models (Sec. 3) and for the MAS implementation (Sec. 4).

ae-mail: Frank.Richtererub.de

This is an Open Access article distributed under the terms of the Creative Commons Attribution-Noncommercial License (http://creativecommons.org/licenses/by-nc/3.0/), which permits unrestricted use, distribution, and reproduction in any noncommercial medium, provided the original work is properly cited. 


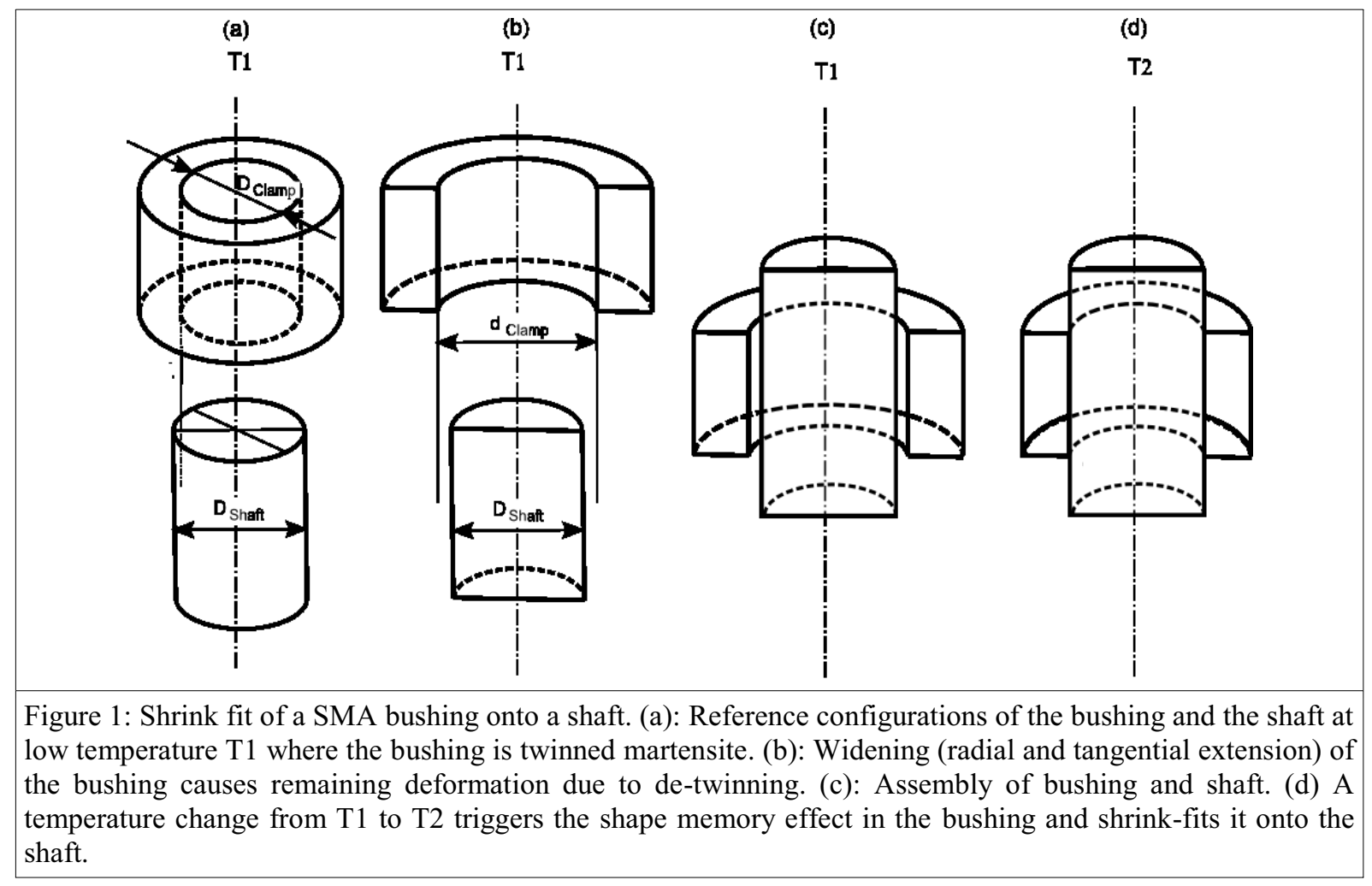

\section{Analytical treatement}

\subsection{Elastic bushing}

Shrink-fitting axisymmetric bodies is a common method in mechanics to achieve a tight fit of a bushing on a shaft. In the reference configuration at low temperature T1, the diameter $D_{\text {shaft }}$ of the shaft is slightly larger than the inside diameter $D_{\text {clamp }}$ of the bushing by a small interference, see Fig. 1 (a). In the conventional shrink fit procedure, the bushing is thermally expanded. In this state the center planes of the shaft and the bushing are vertically aligned and the shrink-fit assembled by cooling, causing a radial contraction of the bushing, thus fixing the bushing onto the shaft. This process is considered by classical mechanics [11]. Employing this thick-wall cylinder theory for identical linear-elastic materials the contact pressure $p$ and the radial distributions of the radial $\left(\sigma_{r r}\right)$ and circumferential $\left(\sigma_{\theta \theta}\right)$ stress in the shaft and the bushing are given as a function of the radial coordinate $r$ by the following set of equations:

$$
p=\frac{Y \cdot \delta \cdot\left(r_{2}^{2}-r_{1}^{2}\right) \cdot\left(r_{3}^{2}-r_{2}^{2}\right)}{2 \cdot r_{2}^{3} \cdot\left(r_{3}^{2}-r_{1}^{2}\right)}
$$

shaft: $\quad \sigma_{r r}=-\frac{p \cdot r_{2}^{2}}{r_{2}^{2}-r_{1}^{2}}+\frac{p \cdot r_{1}^{2} \cdot r_{2}^{2}}{r_{2}^{2}-r_{1}^{2}} \cdot \frac{1}{r^{2}} ; \quad$ bushing: $\sigma_{r r}=\frac{p \cdot r_{2}^{2}}{r_{3}^{2}-r_{2}^{2}}-\frac{p \cdot r_{3}^{2} \cdot r_{2}^{2}}{r_{3}^{2}-r_{2}^{2}} \cdot \frac{1}{r^{2}}$

shaft : $\sigma_{\theta \theta}=-\frac{p \cdot r_{2}^{2}}{r_{2}^{2}-r_{1}^{2}}-\frac{p \cdot r_{1}^{2} \cdot r_{2}^{2}}{r_{2}^{2}-r_{1}^{2}} \cdot \frac{1}{r^{2}} ; \quad$ bushing: $\sigma_{\theta \theta}=\frac{p \cdot r_{2}^{2}}{r_{3}^{2}-r_{2}^{2}}+\frac{p \cdot r_{3}^{2} \cdot r_{2}^{2}}{r_{3}^{2}-r_{2}^{2}} \cdot \frac{1}{r^{2}}$ 
Here, $Y$ is the Young's modulus, $r_{1}$ is the inner radius of the shaft (taken as zero), $r_{2}=D_{\text {shaft }} / 2$ is the outer radius of the shaft and $r_{3}$ is the outer radius of the bushing. Both shaft and bushing have a Poisson's ratio of zero. These theoretical results are represented by the solid black lines in Fig. 2 and Fig. 3.

\subsection{Elastic - plastic bushing}

Next we consider an elastic - ideally plastic bushing. The reasoning behind this step rests in the similarity of the stress-strain curve of a SMA over the loading part with the one for elastic-ideally plastic constitutive behavior (provided the stress does not exceed the transformation stress). The theoretically predicted stress distributions for $\sigma_{r r}$ and $\sigma_{\theta \theta}$ within the bushing are given by

$$
\begin{gathered}
\sigma_{r r}=-\frac{\sigma_{0}}{2}\left(-2 \ln \left(\frac{r}{r_{2}-\delta}\right)+\ln \left(\frac{Y}{\sigma_{0}} \frac{\delta}{r_{2}-\delta}\right)+1\right)+Y \frac{\delta /\left(r_{2}-\delta\right)}{2\left(\frac{r_{3}}{r_{2}-\delta}\right)^{2}} \\
\sigma_{\theta \theta}=\frac{\sigma_{0}}{2}\left(2 \ln \left(\frac{r}{r_{2}-\delta}\right)-\ln \left(\frac{Y}{\sigma_{0}} \frac{\delta}{r_{2}-\delta}\right)+1\right)+Y \frac{\delta /\left(r_{2}-\delta\right)}{2\left(\frac{r_{3}}{r_{2}-\delta}\right)^{2}}
\end{gathered}
$$

wherein $\sigma_{0}$ is the yield stress [12]. These stress distributions are depicted as black dots in Fig. 2 and Fig. 3.

\section{The FEM model}

\subsection{Geometry, discretization}

The geometry of the FEM model is identical for all simulations: the inner radius of the shaft $r_{1}$ equals zero, its outer radius $r_{2}=D_{\text {shaft }} / 2$ equals $5.1 \mathrm{~cm}$. The bushing is limited by its inside diameter $D_{\text {clamp }}$ of $5 \mathrm{~cm}-$ hence an interference $\delta$ of $0.1 \mathrm{~cm}$ - and an outside diameter $r_{3}$ of $7 \mathrm{~cm}$. The bushing is $4 \mathrm{~cm}$ tall, the shaft three times as tall. The region of interest is spatially discretized (horizontal x vertical) into $50 \mathrm{x} 150$ (shaft) resp. $10 \mathrm{x}$ 50 (bushing) elements. The task is simplified by meshing the shaft with axisymmetric stress/displacement elements CAX4 and the bushing with CAX4T elements in ABAQUS TM. This element type features an additional degree of freedom for the temperature.

We employ standard ABAQUS TM material routines to test both the model and the solution strategy for the cases of ideal-elastic and elastic-plastic material properties. In these cases, the shrinking is induced by thermal contraction modeled here by assigning only a radial and tangential thermal expansion coefficient for the bushing, whereas its axial expansion coefficient was set to zero in order to meet the assumption of the classical treatments of a plane stress state from Sec. 2 (Eqs. (2) and (3)).

The FEM simulation strategy is as follows: The thermally extended bushing is assembled with the shaft at high temperature as shown in Fig. 1 (c). Then, the temperature is lowered linearly in time so as to induce thermal contraction of the bushing.

The abovementioned formulas are the simplest ones for shrink-fitting as they refer to materials with initially identical constitutive behavior. Thus, the shaft - which is always modeled as linearly elastic - and the bushing in the elastic and elastic - ideally plastic simulations have a Young's modulus equal to the one taken for martensite (numerical value see Section 4.2). For simplicity, the thermal expansion of the shaft is neglected. 


\subsection{Verification of the numerical model by benchmark tests}

We simulate shrink fitting and compare results to benchmark tests to assure the reliability of the approach. For the classical cases of Sec. 2 the reliability of the FEM model is tested by comparison to Eqs. (2) to (5), see Fig. 2 for the radial and Fig. 3 for the circumferential stress components in shaft and bushing. In these diagrams we refer to the analytical solutions by solid black lines for the ideal elastic case and by black points for the elasticplastic case. The FEM solutions obtained for ideal elastic material are plotted by solid green lines and for the elastic-plastic material by solid blue lines.

Inspection of Figs. 2 and 3 show that the FEM solutions match well the analytical solutions in the bushing. In the shaft the FEM solution for the radial stress $\sigma_{r r}$ shows a deviation (arrow in Fig. 1) that grows with decreasing radial coordinate up to a deviation of $1 / 3$ at zero radius.

The cause for the discrepancy is not precisely known; a finer spatial discretisation of the FEM mesh for the shaft did not modify the results. However, observing much better correspondence to theory when the inside diameter of the shaft is not zero (Fig. 4) suggests that ABAQUS encounters numerical problems when searching for a solution at a radial singularity. It is stressed that the radial displacements as a function of the radius is close to the theoretically predicted one both within the shaft and within the bushing also when the shaft is solid (data not shown here). Keeping in mind the fact that the bushing is of prime concern here, the deviation indicated by the arrow in Fig. 1 is acceptable.

As for the simulation for an elastic - ideally plastic behavior of the bushing, the numerical results (shown in blue in Fig. 2 and Fig. 3) are very close to the ones demanded by Eqs. (4) and (5) (black dots), lending more support to the simulations. It is thus concluded that the numerical model as programmed here represents a sufficiently precise numerical procedure for shrink-fitting.

\section{Simulation of a shrink fit with a shape memory bushing}

\subsection{The MAS model and its FEM implementation}

Crystallographic observations have revealed that SMAs exhibit a layered crystalline structure. The MAS model rationalizes this observation by considering ideal mesoscopic crystal layers in one of the three model phases austenite (A) and two martensitic twin phases (M+, M-) [1-9]. The model derivation adheres strictly to thermodynamics and roots in the idea of a three-well potential energy with minima indicating the stable locations of these three phases. The model yields a constitutive equation for the stress $\sigma$ as a function of external strain $\varepsilon$ and the internal variables $x_{A}, x_{M_{+}}$and $x_{M_{-}}$which express the phase fractions of the model phases,

$$
\sigma=E_{M} \cdot \frac{\varepsilon-\left(x_{M+}-x_{M-}\right) \cdot \varepsilon_{T}}{x_{M+}+x_{M-}+\frac{E_{M}}{E_{A}} \cdot x_{A}} .
$$

The elasticity of the pure phases is expressed by the Young's moduli $E_{M}$ and $E_{A}$ and a martensitic transformation causes a residual strain $\varepsilon_{T}$. According to the MAS model, the phase fractions are implicitly functions of the temporal evolution of the stress-strain field and the temperature. These are determined by a set of rate equations representing an initial value problem (coupled ODE system) solved numerically for given strain and temperature. The numerically stiff feature of this ODE requires a stable integration scheme for robust solution. Here we use an implicit Runge-Kutta scheme of order five with an adaptive time step size called RADAU IIa [13]. The temperature evolution within the specimen is due to latent heat emission and absorption and the according heat exchange with the ambiance. Assuming temperature homogeneity in simple geometries, the temperature can be calculated from the integral energy balance which represents an additional differential equation to be solved along with the rate equations for the phase fractions. Previously, this was accomplished by 
a standalone FORTRAN program [5,8]. An online version of this model considering a SMA wire is illustrated on the internet [14].

ABAQUS/Standard allows user implementations of constitutive equations of state through a FORTRAN subroutine called UMAT (User Material), where the state of the nodal field quantities (here: displacements and temperature) can be accessed. UMAT is called by ABAQUS within its own numerical procedure while striving for a converged solution. Here, we have used the UMAT interface to adopt the standalone implementation of the MAS model into the ABAQUS environment. Further, the user has to provide the Jacobian stiffness matrix which for the MAS model reads

$$
\frac{d \sigma}{d \varepsilon}=\frac{\partial \sigma}{\partial \varepsilon}+\frac{\partial \sigma}{\partial x_{M+}} \cdot \frac{d x_{M+}}{d t} \cdot \frac{d t}{d \varepsilon}+\frac{\partial \sigma}{\partial x_{M-}} \cdot \frac{d x_{M-}}{d t} \cdot \frac{d t}{d \varepsilon}
$$

owing to the dependence of the phase fractions on time $t$ and the identity $x_{A}=1-\left(x_{M_{+}}+x_{M_{-}}\right)[16,17]$ while $\varepsilon_{T}$ is a constant. Further details on the numerics are provided in [15].

\subsection{Implementation of the MAS model for simulating a shrink-fit}

The references listed in the previous section have provided ample justification for the claim that the MAS model has potential for efficient simulations of SMA behavior. The MAS model in its present uniaxial formulation restricts applicable load scenarios to those where uniaxial stress dominates such as loading in wires and trusses [16] as well as pure beam bending [17], and pure torsion [18,15]. A tool for simulating wires and trusses is accessible via an internet page for the convencience of the scientific community [14]. The model also addresses polycrystalline materials $[19,20,15]$. Arbitrary, multiaxial stress/strain states can be addressed by the use of multivariant extension of the MAS model in principle [21], however the FEM implementation of such model is an open task.

As the analytical solution shows that the circumferential (hoop) stress dominates (see Fig. 2 and Fig. 3), shrink fitting is a load case amenable to the MAS model. The MAS-model is implemented here in its version for single crystals exclusively for the hoop direction, retaining elasticity for the other ones. The numerical realization of this characteristic lies in first defining elasticity for the entire stress tensor, then modifying the constitutive behavior for the circumferential direction, all in one UMAT.

In the reference state (Fig. 1a), the bushing is composed of equal amounts of the martensite variants $\mathrm{M}+$ and $\mathrm{M}$-. The radial widening (Fig. 1b) is achieved by a radial load exerted on the face of the inner diameter of the bushing which effects load-induced $M-\Rightarrow M+$ transitions (de-twinning) till a pure $\mathrm{M}+$ state is attained. In this state the bushing is assembled with the shaft (Fig. 1c) and subsequently heated up to trigger the shape memory effect, i.e., the transformation of martensite to austenite. All model parameters for the SMA are collected in Table 1 .

Table 1. Model parameters for SMA (taken from $[16,17])$.

\begin{tabular}{|l|l|}
\hline Young's modulus of austenite & $71.1 \mathrm{GPa}$ \\
\hline Young's modulus of martensite & $30.9 \mathrm{GPa}$ \\
\hline transformation strain & $4.4 \%$ \\
\hline linear coefficient in the free energy of unstrained austenite & $-396000 \mathrm{~J} /(\mathrm{g} \cdot \mathrm{K})$ \\
\hline free energy of unstrained austenite at T $=0 \mathrm{~K}$ & $0.115 \mathrm{GJ} / \mathrm{kg}$ \\
\hline activation volume & $5 \cdot 10^{-23} \mathrm{~m}^{3}$ \\
\hline density & $6500 \mathrm{~kg} / \mathrm{m}^{3}$ \\
\hline specific heat capacity & $450 \mathrm{~J} /(\mathrm{kg} \cdot \mathrm{K})$ \\
\hline
\end{tabular}

The temperature variation is treated in two different ways: First, temperature is homogeneously controlled by a linear function in time (quasistatic simulation, Sec. 4.2.1). In this case the energy balance controlling the 
temperature field in the bushing is not considered. Second, the shrink fit problem is fully coupled with the temperature field (Sec. 4.2.2). In this case, the surface temperatures of the bushing are controlled by Dirichlet conditions in time and the evolution of the temperature are controlled by the energy balance within ABAQUS.

\subsubsection{Quasistatic simulation}

This procedure prescribes the rate of temperature rise which was set uniform for all (i.e., also internal) elements. Heat exchange with the ambiance and internal heat flux are neglected. The result arrived at for the temperature is referred to as the state variable SDV4 to clearly distinguish it from the temperature in the fully coupled simulation (see section 4.2.2). The data so obtained are shown in turquoise in Fig. 2 and Fig. 3.

\subsubsection{Thermomechanically coupled simulation}

The physically sound approach, benefitting from the capability of the MAS model and the finite-element method, is the thermomechanically coupled procedure (ABAQUS TM command COUPLED TEMPERATUREDISPLACEMENT). A heating rate is applied to the external nodes only. In addition to this imposed temperature rise latent heat is emitted resp. absorbed throughout the bulk of the bushing, but this contribution generates locally different values. Upon convergence, the stresses and the latent heat production due to phase transformations are returned from UMAT to ABAQUS. Thermomechanical coupling is achieved by ascribing the actual specific latent heat production to the ABAQUS variable RPL within UMAT, hence serving as a production term to the global energy balance. Within ABAQUS, the internal heat flux is expressed by Fourier's law and the surface heat flux by Dirichlet or Neumann boundary conditions, respectively. The respective results are shown in red in Fig. 2 and Fig. 3.

\subsection{Discussion of the SMA shrink fit}

The resulting radial stress distributions are shown in Fig. 2 and Fig. 3 along with all previous results. The stress profiles for the quasistatic SMA simulation bears a strong resemblance to the elastic - ideally plastic one whereas the ones originating from the thermomechanically coupled one deviate strongly. Strict adherence to the elastic- ideally plastic case cannot be expected (even if the stress in the SMA simulation does not exceed the transformation stress) as the MAS model does not preserve the volume while plasticity does.

It is stressed that the results for the SMA bushing show the conditions at maximum temperature while those for the elastic and elastic-ideally plastic one show the conditions prevailing when the assembly is back at its initial temperature. The stress state within the assembly and the grip between shaft and bushing is preserved as long as the temperature does not fall below the maximum martensite start temperature present in the bushing. If it does, the austenite-martensite transformations are triggered with a preference for $\mathrm{M}+$ due to the hoop stresses still present in the bushing. This ongoing transformation process reduces the hoop stresses and eventually yields an unstressed assembled bushing without gap. This transformation is initiated at approx. $390 \mathrm{~K}$ in the above quasistatic simulation; a temperature far above common usage for bushings. Thus, the numerical values used in the present publication need to be adjusted.

As transformation rates are affected by temperature and stress, enlightening aspects of SMA behavior can be expected when tracking certain quantities as a function of both temperature (state variable SDV4 in the quasistatic simulation resp. temperature in the coupled simulation) and radial position (i.e., stress as shown in Fig. 2 and Fig. 3). This intricate behavior is revealed by Fig. 5. Here, data are presented for the austenite fraction as a function of temperature (SDV4) and radial position within the bushing. The color coding is red for the innermost element just above the center plane and green for the outermost one. Data referring to the quasistatic simulation are shown as solid lines whereas those for the fully coupled one are depicted as colored circles with a black rim. The results from the coupled simulation are additionally shown vs. temperature as blue dots which are seen to deviate only slightly from the result of state variable SDV4.

Fig. 5 reflects the Clausius-Clapeyron relation for SMA by a shift of the austenite start and finish temperatures with hoop stresses. Evidently, the common definition of austenite start temperature $\left(\mathrm{A}_{\mathrm{s}}, 10 \% \mathrm{~A}\right)$ and austenite finish temperature $\left(\mathrm{A}_{\mathrm{f}}, 90 \% \mathrm{~A}\right)$ yields data that depend on the radial coordinate. According to Fig. 5, $\mathrm{A}_{\mathrm{s}}$ is quite uniform along the radial coordinate for the quasistatic simulation whereas $A_{f}$ has a wide span through the bushing thickness. Instead, the temperature range for $A_{s}$ is far greater in the coupled simulation whereas $A_{f}$ is 
quite small. In the final state (Fig. 3) this stress is equal at the innermost and outermost node, but this observation does not hold for the entire process. Thus, hereditary phenomena contribute to a various extent throughout the simulation.

The radial stress profile over the cross-section in the assembled state is depicted in Fig. 6. For a clear visual impression, the axisymmetric elements are swept over $180^{\circ}$. Obviously, axial stress gradients emerge and it is reminded that Fig. 2 and Fig. 3 show data only on the center planes.

\section{Conclusions and future work}

The results discussed evince potential of the method employed for industrial usage in cases of design studies of devices which incorporate the shape memory effect and add to the versatility of the MAS model. The shrink-fit problem of a bushing, though a simple device, reveals many aspects of fully-coupled FEM studies and thereby serves as an adequate test case of the model. Such bushings are temperature-activated and conveniently used as they offer the possibility for repeated application and release solely by the application of heat. The only aspect to be noted is the minimum service temperature. Whenever cooled below it, the austenite will decompose into the martensite variants and loosen its grip.

Further, distinctly different results are obtained when thermomechanical coupling is accounted for. The totally different stress state compared to all prior simulations proves the necessity for incorporation of all relevant physical effects.

The authors appreciate financial support by the German Research Foundation (grant KA 2304/2-1).

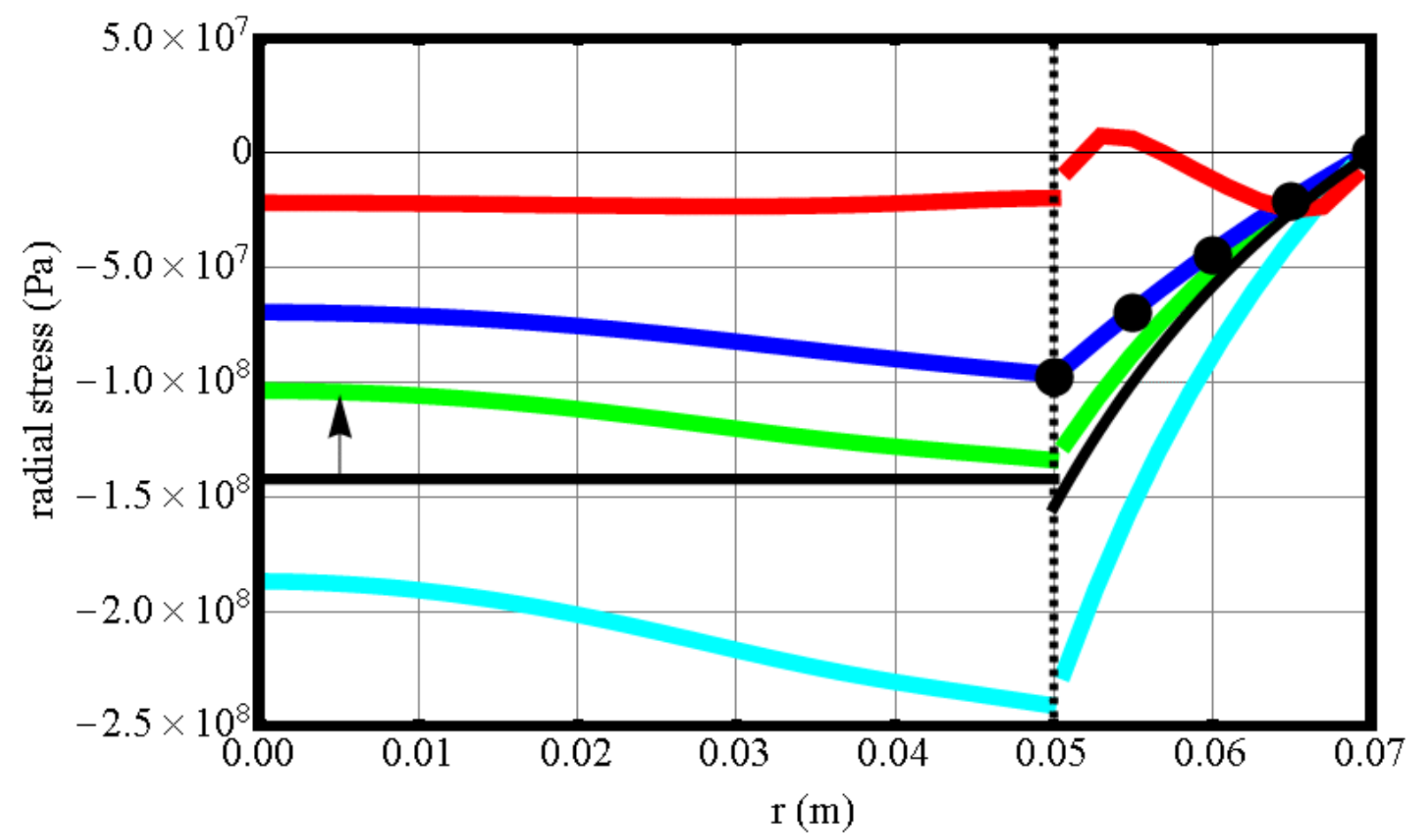

Fig. 2. Radial stress distribution along the radial coordinate for all FEM simulations for different bushing constitutive behaviors (green: elastic, blue: elastic - ideally plastic, turquoise: SMA quasistatic, red: SMA thermomechanically coupled) and the theoretical formulas (elastic: black solid lines (Eqs. (2) and (3)), elastic - ideally plastic: black dots (Eqs. (4) and (5)). Dashed black vertical line at $r=0.05 m$ delineates shaft from bushing (original dimension). Initial dimensions: shaft in $0 \leq r \leq 0.051 \mathrm{~m}$, bushing in $0.05 m \leq r \leq 0.07 \mathrm{~m}$. Arrow indicates deviation between theory and numerics within the shaft in the elastic case. 


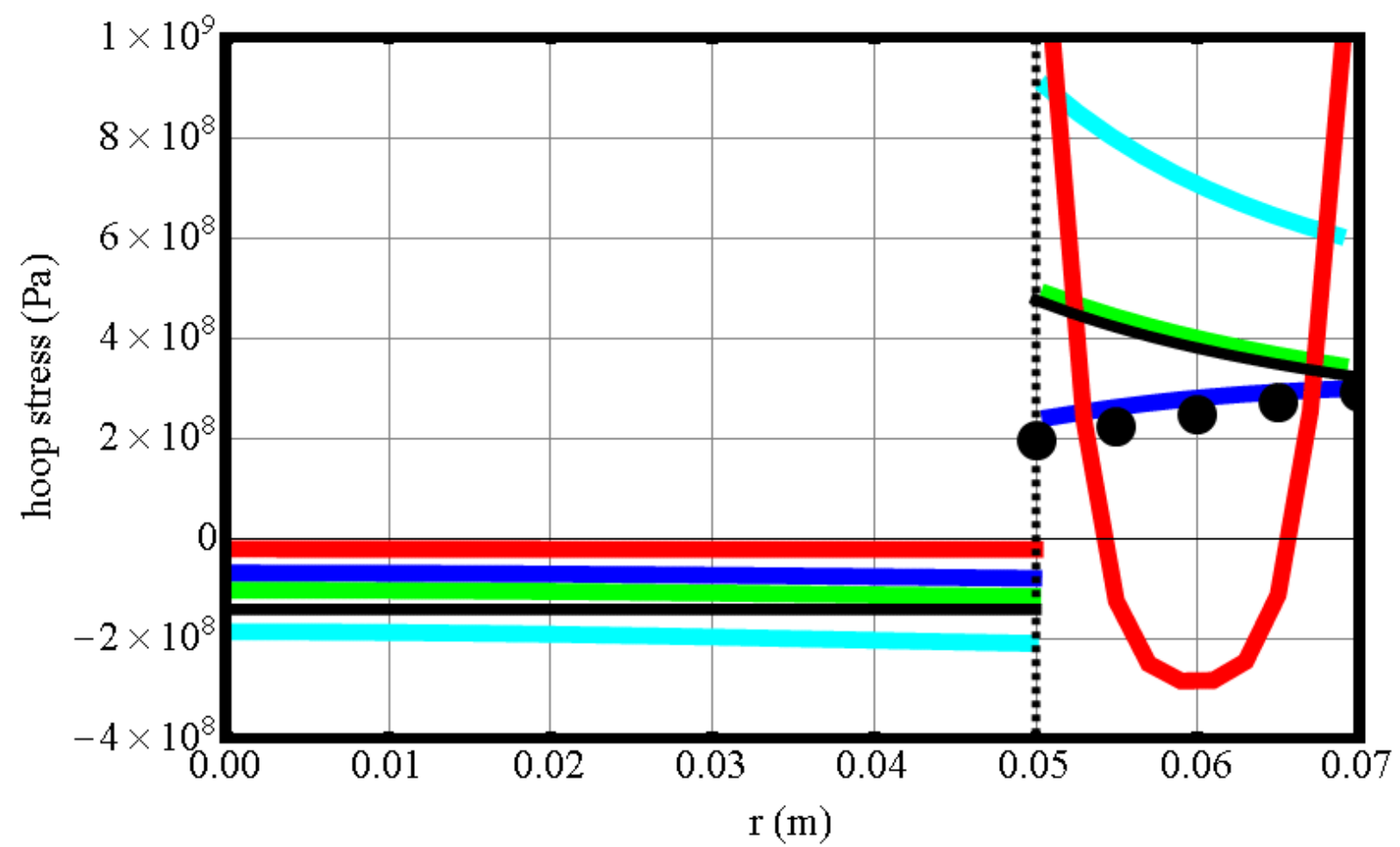

Fig. 3. Circumferential stress distribution along the radial coordinate for all simulations and the theoretical formulas. Color coding and initial dimensions see Fig. 2.

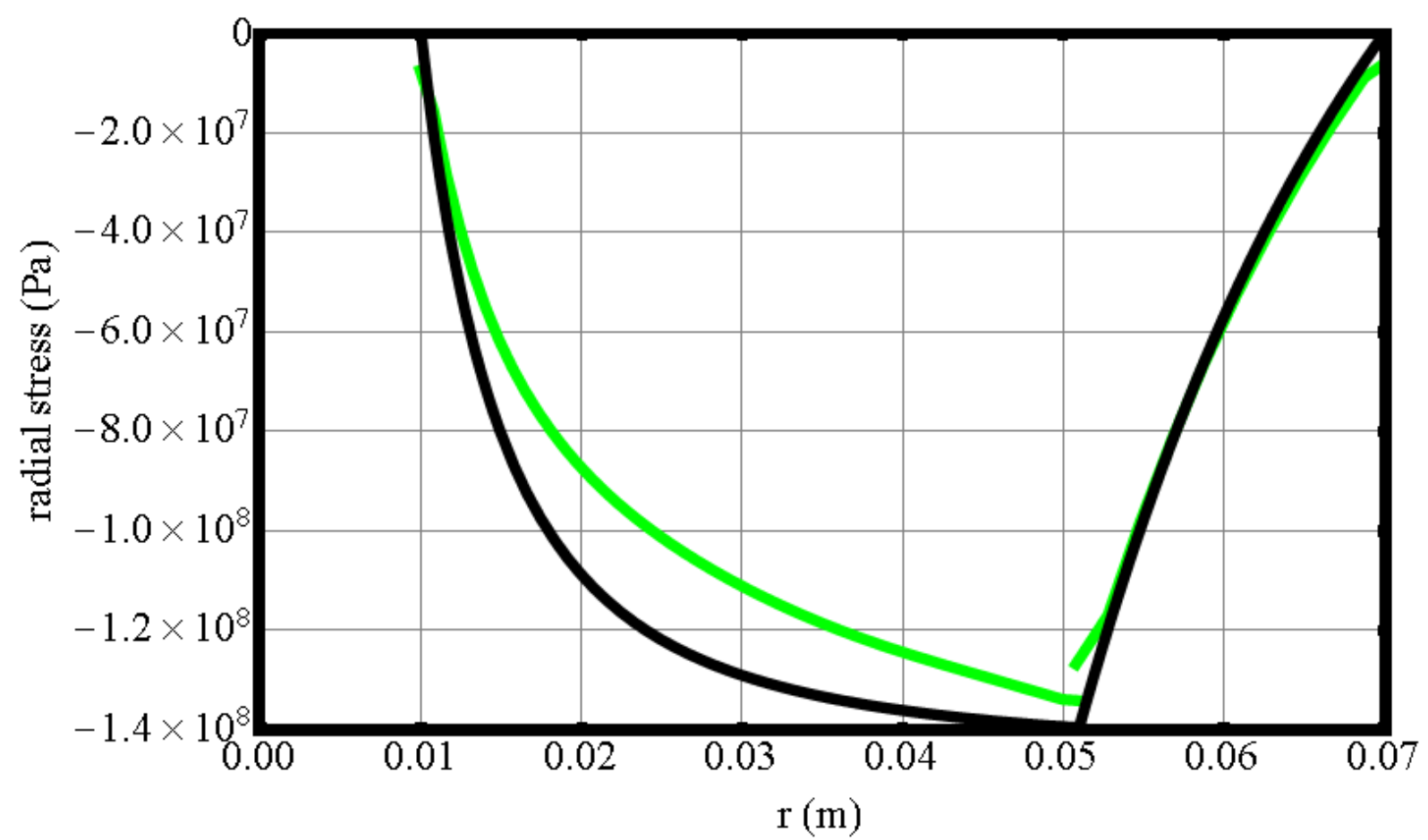

Fig. 4. Radial stress distribution along the radial coordinate for a simulation of shrink-fitting an elastic bushing with an inner radius of $1 \mathrm{~cm}$ onto an elastic shaft. Theoretical results (Eq. (2)): black, numerical data: green. 


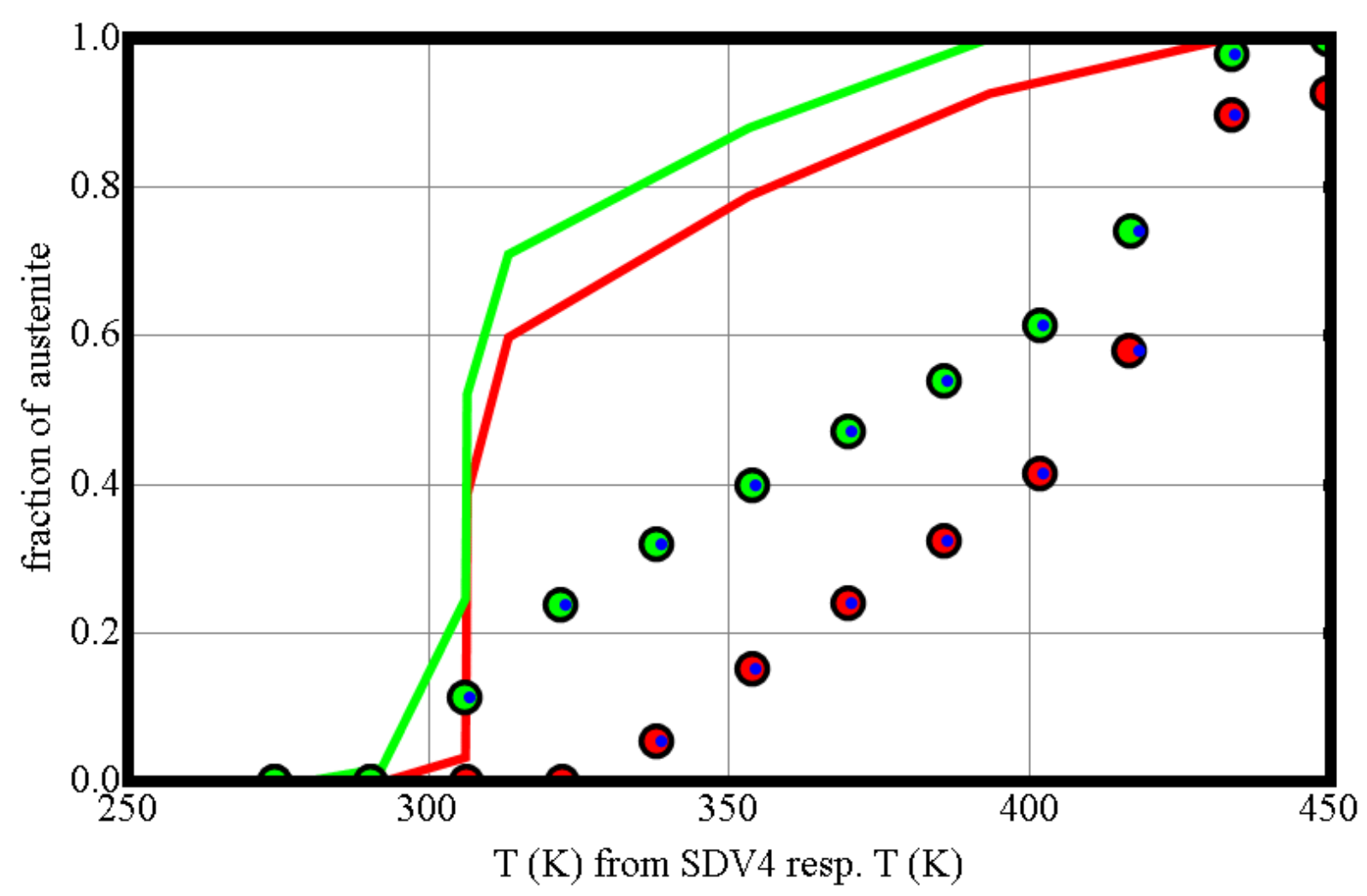

Fig. 5. Fraction of austenite as a function of two temperature axes (see text) and radial coordinate (red for the innermost element just above the center plane and green for the outermost one). Solid lines: quasistatic simulation, dots with black rim: coupled simulation; both plotted vs. temperature from state variable SDV4. Blue dots: coupled simulation, but plotted vs. temperature arising in this simulation (see section 4.2.2).
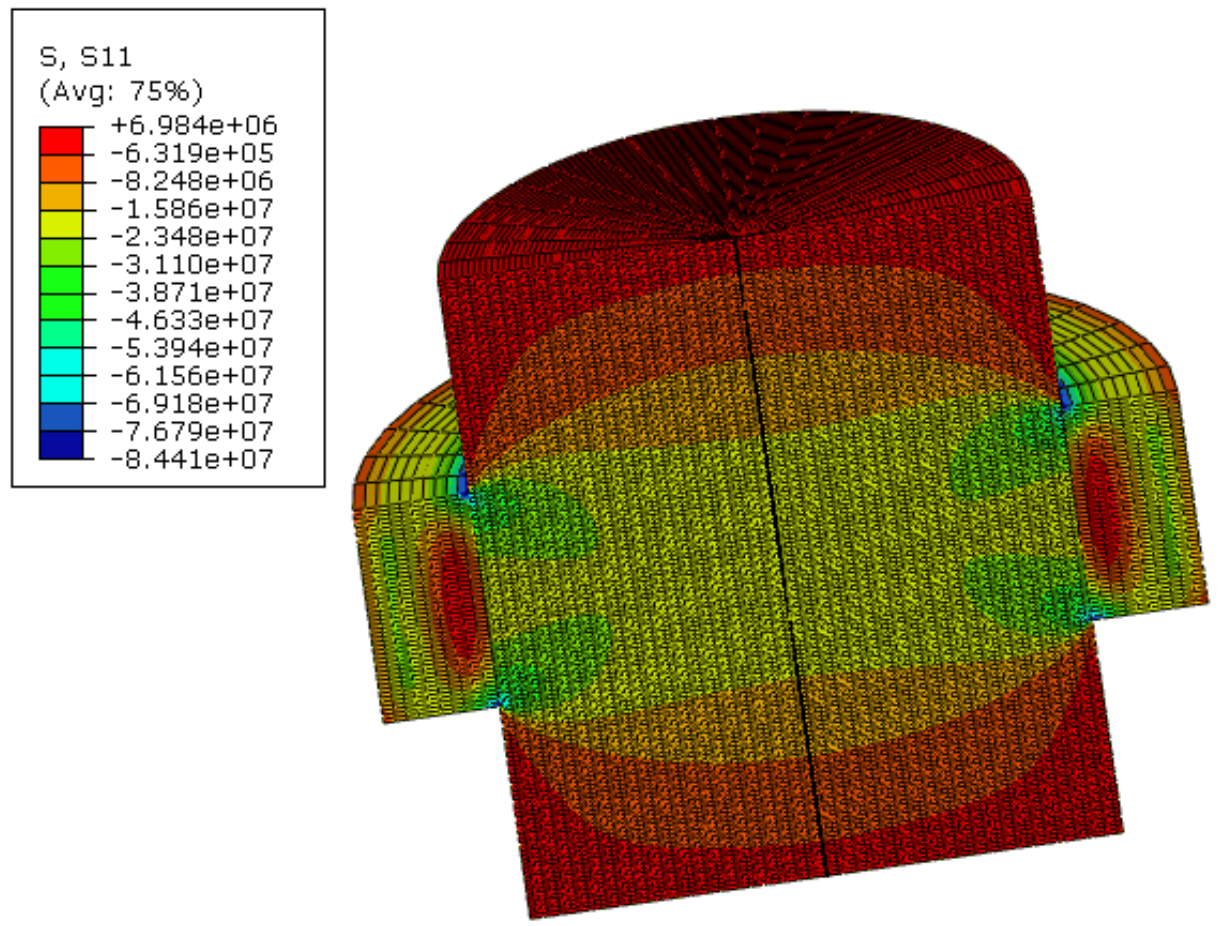

Fig. 6. Cut-away view of the shrink fit in the assembled state showing the spatial distribution of the radial stress distribution over the cross-section. Note the variation of the radial stress with axial position. 


\section{References}

[1] I. Müller, Arch. Rat. Mech. Anal. 70(1), 61-77 (1979)

[2] M. Achenbach, I. Müller, J. de Phy. Colloque C4, Supplement au no 12, 163-167 (1982)

[3] M. Achenbach, I. Müller, Arch. Mech. 37, 573-585 (1985)

[4] M. Achenbach, T. Atanackovic, I. Müller, Int. J. Solids Struct. 22, 171-193 (1986)

[5] M. Achenbach, Int. J. Plast. 5(4), 371-395 (1989)

[6] M. Achenbach, "Simulation des Spannungs-Dehungs-Temperatur Verhalten von Legierungen mit Formerinnerungsvermögen”, Dissertation, Technische Universität Berlin, Germany, 1987

[7] S. Seelecke, Cont. Mech. Therm. 9, 165-173 (1997)

[8] S. Seelecke, I. Müller, Math. Comp. Mod. 34, 1307-1355 (2001)

[9] S. Seelecke, I. Müller, Appl. Mech. Rev. 57(1), 23-46 (2004)

[10] F. Richter, "Finite-element-simulations of polycrystalline shape memory alloys", Smart Structures and Materials \& Nondestructive Evaluation and Health Monitoring 2008, San Diego, USA, March 09-13, 2008

[11] G. Lamé, B. Clapeyron, Mémoire sur l'équilibre intérieur des corps solides homogènes, J. reine angew. Math. 7, 150-169, 237-252, 381-413 (1831)

[12] U. Gamer, R.H. Lance, Forsch. Ing.-Wes. 48(6), 192-198 (1982)

[13] E. Hairer, G. Wanner, Solving Ordinary Differential Equations II: Stiff and Differential-Algebraic Problems (Springer Series in Computational Mathematics 14, 2nd ed., Springer, Berlin, 1996)

[14] Interactive webpage for the simulation of shape memory wires, www.smaterial.com

[15] F. Richter, O. Kastner, G. Eggeler, accepted by J. Mat. Eng. Perf., special issue containing contributions from the "Shape Memory and Superelastic Technology Conference", Stresa, Italy, September 21-25, 2008

[16] J.P. Frautschi, "Finite Element Simulations of Shape Memory Alloy Actuators in Adaptive Structures", M.Sc. thesis, North Carolina State University, USA, 2003

[17] Q. Li, "Modeling and Finite Element Analysis of Smart Materials", Ph.D. Thesis, North Carolina State University, USA, 2006

[18] O. Heintze, O. Kastner, H. Sahota, S. Seelecke S., „The role of thermomechanical coupling in the dynamic behavior of shape memory alloys", IUTAM Symposium on Smart Structures and Structronic Systems, Proceedings of the IUTAM Symposium, Magdeburg, Germany, Sept 26-29, 2000, edited by U. Gabbert and H.S. Tzou (Springer, 2001), p. 145-152

[19] O. Heintze, "A computationally efficient free energy model for shape memory alloys - experiments and theory", Ph.D. Thesis, North Carolina State University, USA, 2004

[20] O. Heintze, S. Seelecke, Mat. Sci. Eng. A, 481-482, 389-394 (2008)

[21] O. Kastner, F. Richter, G. Eggeler, "Multivariant formulation of the thermomechanically coupled MüllerAchenbach-Seelecke-model for shape memory alloys", ASME 2009 Conference on Smart Materials, Adaptive Structures and Intelligent Systems, Oxnard, USA, September 21-23, 2009 